\title{
Limited role for ASC and NLRP3 during in vivo Salmonella Typhimurium infection
}

\author{
Hanna K De Jong ${ }^{1,2^{*}}$, Gavin CKW Koh ${ }^{2,3}$, Miriam HP van Lieshout ${ }^{1,2}$, Joris JTH Roelofs ${ }^{4}$, Jaap T van Dissel ${ }^{5}$, \\ Tom van der Poll ${ }^{1,2}$ and $W$ Joost Wiersinga ${ }^{1,2}$
}

\begin{abstract}
Background: The inflammasome is an intracellular protein complex triggered by exposure to intracellular pathogens, its components or other endogenous proteins. It leads to the activation of and subsequent release of proinflammatory cytokines such as IL-1 $\beta$ and IL-18. S. Typhimurium is a Gram-negative intracellular bacterium, which is known to trigger inflammasome assembly via recognition by the cytosolic receptors, NLRP3 and NLRC4 (which act via the adaptor protein, ASC) to induce cell death and cytokine release. We sought to characterize the role of ASC and NLRP3 in two different murine models (typhoid and colitis) of systemic Salmonella infection.
\end{abstract}

Results: Release of the inflammasome cytokine IL-18 was hampered in $\mathrm{AsC}^{-/-}$but not N/rp3 ${ }^{-/-}$mice (background C57BL/6) during S. Typhimurium infection. Unexpectedly, neither ASC nor NLRP3 played a significant role in host defense against $S$. Typhimurium infection, as reflected by equal bacterial counts in $\mathrm{WT}, \mathrm{Asc}^{-1-}$ and ${\mathrm{N} / r p 3^{-/-}}_{\text {mice }}$ at all time points, in both the typhoid and colitis models. Proinflammatory cytokine levels (TNF-a, IL-6) and the extent of hepatic and splenic pathology did not differ between groups in the typhoid model. In the colitis model small differences were seen with regard to splenic and hepatic inflammation, although this was IL-18 independent.

Conclusions: IL-18 release was reduced in $\mathrm{Asc}^{-/-}$but not $\mathrm{N} / r p 3^{-/-}$mice during S. Typhimurium infection. Despite this reduction, bacterial counts, cytokine levels and histological inflammation did not differ between wild-type and knockout mice in either model. Our results reveal a limited role for ASC and NLRP3 during in vivo S. Typhimurium infection despite its role in cytokine maturation.

Keywords: Inflammasomes, Salmonella Typhimurium, Host-pathogen interactions

\section{Background}

Invasive Salmonellosis is a global burden with more than 100 million cases per year resulting in over 350,000 deaths [1-3]. Salmonella enterica infections, which are the Gram-negative intracellular bacteria responsible for this burden, can result in diverse clinical manifestations. Food-borne non-typhoidal Salmonella (NTS), caused by serovar Typhimurium or Enteriditis, has recently emerged as a prominent cause of bloodstream infection, primarily in African adults and children, with an associated case fatality of up to $25 \%$. HIV, malaria, and malnutrition being

\footnotetext{
*Correspondence: H.K.deJong@amc.uva.nl

'Department of Internal Medicine, Division of Infectious Diseases and Center for Infection and Immunity Amsterdam (CINIMA), Academic Medical Center, Meibergdreef 9, Room G2-132, 1105, AZ, Amsterdam, the Netherlands

${ }^{2}$ Center for Experimental and Molecular Medicine (CEMM), Academic Medical Center, Meibergdreef 9, Room G2-132, 1105, AZ, Amsterdam, the Netherlands

Full list of author information is available at the end of the article
}

important risk factors [2]. Typhoid or enteric fever, caused by the exclusively human serovar Typhi or Paratyphi A, is a bacteremic disease that can result into intestinal perforation, peritonitis, encephalopathy myocarditis and hemodynamic shock [3-5]. Antimicrobial resistance for all Salmonella enterica infections is widespread [6]. The variations in the clinical features of infection with these intracellular pathogens relate to differences in the interaction between different Salmonella serovars and the host [3]. A better understanding of host-pathogen interactions in invasive Salmonellosis could explain these diverse clinical manifestations and potentially lead to new therapeutic strategies in order to decrease the considerable morbidity and mortality.

Salmonella spp. are recognized by pattern recognition receptors (PRR), via conserved motifs termed 'pathogenassociated-molecular-patterns', resulting in activation of signaling pathways that initiate the inflammatory response 
[3,7-9]. The two most important families of PRRs are the membrane-bound Toll-like receptors (TLRs) and the cytosolic Nod-like receptors (NLRs). Pro-inflammatory cytokines such as interleukin (IL)-6, IL-1 $\beta$ (also called IL-1 F2), IL-18 and tumor necrosis factor (TNF)- $\alpha$ are released during early Salmonella infection. By contrast, interferon (IFN)- $\gamma$ secretion (triggered by IL-18) plays a central role in the control of persistent infection by affecting the extent of macrophage activation [10-16]. Salmonella spp. expresses multiple pathogen-associated-molecularpatterns, most notably type 3 secretion systems (T3SS), flagella, fimbriae, lipopolysaccharide (LPS) and bacterial DNA $[3,4]$. TLR activation induces the synthesis of pro-IL$1 \beta$ and pro-IL-18, upon which a second signal is provided by the activation of the intracellular inflammasome and caspase- 1 leading to IL-1 $\beta$ and IL-18 processing [8,17].

The role of the inflammasome in the recognition of Salmonella spp. has been studied extensively using in vitro and in vivo models [9]. Best studied NLRs in the recognition of Salmonella are the pyrin domain containing-3 (NRLP3) and the CARD domain-containing protein-4 (NLRC4), which form inflammasome complexes consisting of caspase- 1 and the adaptor protein apoptosisassociated speck-like protein containing a CARD (called PYCARD or ASC) [18]. Both the inflammasome receptors activate caspase- 1 in response to $S$. Typhimurium infection together with endogenous signals, and recruit ASC and caspase- 1 into a single cytoplasmatic focus, which subsequently serves as the site for pro-IL-1 $\beta$ processing [18]. Knockout mice lacking functional casp1, casp1casp11 double knockout mice, or mice deficient in the end product of inflammasome activation (viz., IL-1 $\beta$ and IL-18), do have higher bacterial loads and succumb earlier upon infection with $S$. Typhimurium $[19,20]$. It was therefore hypothesized that $\mathrm{Nlrp3}^{-/-}$(and $\mathrm{Asc}^{-/-}$ mice especially), having lower levels of inflammasomeprocessed cytokines, would succumb earlier to infection with $S$. Typhimurium. On contrary, mice deficient in NLRC4, NLRP3 or ASC were still able to clear S. Typhimurium as efficiently as control mice $[18,21]$. An explanation given for this discrepancy in literature was that intracellular Salmonella can also induce a Salmonella pathogenicity island (SPI)-1 independent form of lytic cell death via caspase-11 [20]. However, casp11 deficient mice have a phenotype comparable to wild-type (WT) mice, showing a role for caspase-11 in the absence of caspase-1-mediated immunity [20]. Furthermore, pregrowth conditions could have also led to this unexpected outcome, where conditions that favor SPI-1 expression (log-phase bacteria) show minimal roles for the inflammasome, while SPI-2 growth conditions (stationary phase bacteria) show a more significant role for caspase-1 during Salmonella infection [9]. Additionally, NLRC4-dependent bacterial clearance is independent of NLRP3, ASC, IL-18, and IL-1R, which could perhaps be an explanation for the unexpected phenotype of ASC-deficient animals [17]. By contrast, cytokine processing that correlates with the formation of this ASC/caspase-1 focus, ASC-independent inflammasomes containing un-processed but active caspase- 1 is also able to initiate rapid cell death [22]. Yet, the relative importance of inflammasome-mediated cytokine maturation and pyroptosis during $S$. Typhimurium infection still remains to be investigated.

In this study, we examined the in vivo relevance of ASC and NLRP3 during Salmonella infection using two clinically relevant experimental models. All mice received $S$. Typhimurium orally and either developed a typhoid-like disease (typhoid model) or, when pretreated with streptomycin, developed a non-typhoid salmonellosis (colitis model). We focused on bacterial growth, systemic cytokine release and organ pathology caused by $S$. Typhimurium using log-phase bacteria.

\section{Methods}

\section{Ethics statement}

Experiments were carried out in accordance with the Dutch Experiment on Animals Act and approved by the Animal Care and Use Committee of the University of Amsterdam (Permit number: DIX102113, DIX102114).

\section{Mice}

Eight- to 12 -week-old male $\mathrm{Asc}^{-/-}$and $\mathrm{Nlrp3}^{-/-}$mice, backcrossed 9 times to a C57BL/6 genetic background, were generated as described [23] and bred in the animal facility of the Academic Medical Center (Amsterdam, the Netherlands). Pathogen-free C57BL/6 WT mice were purchased from Charles River Laboratories Inc. (Maastricht, the Netherlands). Age- and sex-matched animals were used in all experiments and were housed in rooms with a controlled temperature a 12-h light-dark cycle, and were acclimatized for 2 weeks before the experiments. All experimental procedures and animal handling were done during the light cycle.

\section{Experimental infection and design}

For preparation of the inoculum Salmonella Typhimurium strain 14028 was used (ATCC, LGC Standards $\mathrm{GmbH}$, Wesel, Germany). Stock bacteria were streaked from frozen aliquots into $50 \mathrm{ml}$ Luria broth for overnight incubation at $37^{\circ} \mathrm{C}$ in a $5 \% \mathrm{CO}_{2}$ incubator. Thereafter, a $1 \mathrm{ml}$ portion for 1:50 dilution was transferred to fresh Luria broth and grown for approximately $2 \mathrm{~h}$ to mid-logarithmic phase (OD 0.4). Bacteria were harvested by centrifugation at $1500 \times g$ for $10 \mathrm{~min}$, washed and resuspended in sterile isotonic Hank's Buffered Salt Solution (HBSS) at a concentration of $10^{7} \mathrm{cfu} / \mathrm{ml} \mathrm{S}$. Typhimurium. Inoculum size was verified retrospectively by serial 10 -fold dilutions on blood agar (BA). Two different Salmonella 
infection models were used: mice were starved for $12 \mathrm{~h}$ (typhoid model) or pre-treated with streptomycin (Rotexmedica GmbH, Trittau, Germany) $7.5 \mathrm{mg} / 100 \mu \mathrm{l}$ HBSS per os (colitis model), and inoculated orally with $10^{6} \mathrm{cfu} / \mathrm{ml}$ S. Typhimurium in $100 \mu \mathrm{L}$ HBSS as described previously [24-26]. At 2 and 5 days after infection (typhoid model) and 4 days (colitis model), mice were anesthetized with Hypnorm (Janssen Pharmaceutica, Beerse, Belgium) and midazolam (Roche, Basel, Switzerland), and then sacrificed by bleeding from the inferior vena cava. Blood was drawn into heparinized tubes and organs (Mesenterial lymph nodes (MLN), liver, spleen) were harvested and homogenized at $4{ }^{\circ} \mathrm{C}$ in four volumes of sterile saline using a tissue homogenizer (Biospec Products, Bartlesville, UK). Death was confirmed by cutting the diaphragm. Bacterial loads were determined by serial ten-fold dilutions on $\mathrm{BA}$ incubated at $37^{\circ} \mathrm{C}$ for $16 \mathrm{~h}$. Homogenates were centrifuged at $1500 \times g$ at $4^{\circ} \mathrm{C}$ for $10 \mathrm{~min}$, and supernatants were stored at $-20^{\circ} \mathrm{C}$ pending assay.

\section{Assays}

IL-18 (Medical and Biological Laboratories, Nagoya, Japan), IL- $1 \beta$ and TNF- $\alpha$ (R\&D, Minneapolis, MN) concentrations were measured by enzyme-linked immunosorbent assays. IFN- $\gamma$, MCP-1, IL-6, IL-10 and IL-12 were measured by cytometric bead array (BD Biosciences, San Jose, CA) in accordance with the manufacturer's recommendations. AST, ALT and LDH were measured in plasma with spectrophotometry (Roche Diagnostics).

\section{Histology}

Liver, spleens, MLN, terminal ileum and cecum were harvested after sacrifice, fixed in $10 \%$ formalin and embedded in paraffin for histology. Sections of $4 \mu \mathrm{m}$ were stained with haematoxylin-eosin, and read by a pathologist who was blinded to the groups. To score liver inflammation and damage, the entire slide surface was analyzed with respect to the following parameters: area of liver with parenchymal inflammation, necrosis and/or abscess formation, portal inflammation and thrombus formation. Each parameter was graded on a scale of 0 to 4 (0: absent; 1 : mild; 2: moderate; 3 : severe; 4 : very severe). Thrombi were scored as follows: 0: no thrombi; 1 : 1-4 thrombi; 2 : 5-9 thrombi; 3 : 10-15 thrombi; 4: more than 15 . The total liver inflammation score was expressed as the sum of the scores for each parameter, the maximum being 16. Spleen sections were scored for inflammation, necrosis/abscess formation, and thrombus formation using the scales given above. The maximum total spleen inflammation score was 12. In both mouse models the cecum, colon and terminal ileum were assessed for neutrophil infiltration, edema and disruption of the epithelium to determine whether there was a colitis or gut infection present as is expected in the colitis model (streptomycin pre-treatment group).

\section{Statistical analysis}

Values are expressed as mean with standard error of the mean unless stated otherwise. Differences between groups were analyzed by Mann-Whitney U test. These analyses were performed using GraphPad Prism version 5.0, GraphPad Software (San Diego, CA). Values of $\mathrm{p}<$ 0.05 were considered statistically significant.

\section{Results}

\section{Murine typhoid model for invasive salmonellosis}

In order to study the in vivo role of ASC and NLRP3 in invasive salmonellosis, we used a murine typhoid model for severe Salmonella infection [4]. Mice were infected with S. Typhimurium strain S14208 per os. This model mimics the pathology of untreated patients presenting with severe typhoid fever and is characterized by advanced liver and spleen inflammation and capillary thrombus formation in the Peyer's patches, which may lead to hemorrhage, necrosis, ulceration and intestinal perforation [27]. The onset of clinical symptoms and the end stage of disease were dependent on the dose administered. Our pilot experiments showed that an oral dosage of $10^{6}$ CFU S. Typhimurium resulted in a mortality rate of over 90\% (LD90) within 2 weeks. When the dosage was increased to $10^{8}$ CFU S. Typhimurium most mice (LD75) died within a week. When mice became clinically ill (as indicated by ruffled fur, behavioral changes and decreased activity) bacterial counts in blood had increased dramatically from $10^{1}$ to $10^{5} \mathrm{cfu} / \mathrm{ml}$ at 2 and 5 days post-infection respectively, with a marked rise in proinflammatory cytokines, most notably TNF- $\alpha$, IFN- $\gamma$ and IL- 6 , in the systemic compartment.

\section{IL-18 is elevated during $S$. Typhimurium infection in WT mice but completely abolished in $\mathrm{Asc}^{-/-}$mice}

Inoculation of WT mice with $S$. Typhimurium resulted in a rapid increase in IL-18 plasma concentrations in the typhoid model (from $162 \mathrm{pg} / \mathrm{ml}$ at day 2, to $627 \mathrm{pg} / \mathrm{ml}$ 5 days post infection; $\mathrm{p}=0.02$ ), corresponding with inflammasome activation (Figure 1A). In $\mathrm{Asc}^{-/-}$mice IL-18 remained undetectable during the course of infection (Figure 1A). Nlrp3 $3^{-/-}$mice, however, expressed equal plasma levels of IL-18 after infection at all time points when compared to WT mice (Figure 1E), indicating that IL-18 production is independent of NLRP3 during experimental $S$. Typhimurium infection in vivo. IL-1 $\beta$ levels remained undetectable in plasma from WT, $\mathrm{Asc}^{-/-}$and $\mathrm{Nlpr}^{-/-}$mice after infection with $S$. Typhimurium (data not shown).

$\mathrm{Asc}^{-/-}$and $\mathrm{Nlrp3}^{-/-}$mice display equal bacterial counts in all organs upon infection with $S$. Typhimurium

Having established that IL-18 is upregulated during murine $S$. Typhimurium infection, we infected WT, $\mathrm{Asc}^{-/-}$and $\mathrm{Nlrp3}^{-/-}$mice with $\mathrm{S}$. Typhimurium (inoculum $10^{6} \mathrm{cfu}$, typhoid model) and sacrificed them after 2 and 


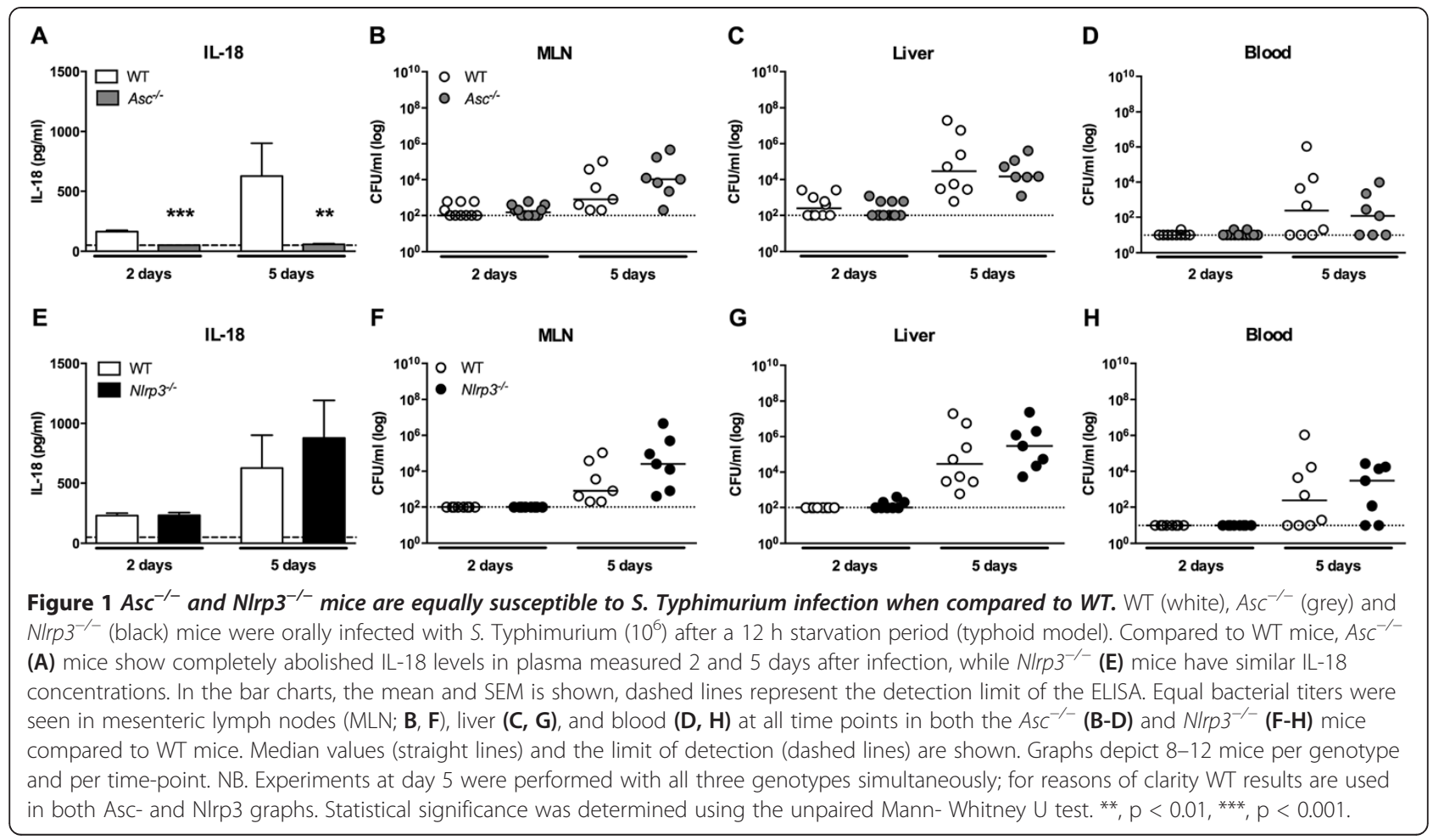

5 days (i.e., before the first deaths occurred) to determine the bacterial loads in MLN (the primary site of the infection once invading bacteria have penetrated the mucosal barrier), liver, spleen and blood in order to evaluate bacterial loads and dissemination to distant body sites. Relative to WT mice, both $\mathrm{Asc}^{-/-}$and $\mathrm{Nlrp}^{-/-}$mice displayed equal bacterial loads in MLNs, liver, spleen and blood at 2 and 5 days after infection (Figure 1B-D, F-H; spleen not shown).

\section{Systemic inflammatory non-inflammasome related} cytokine release during in vivo $S$. Typhimurium infection is ASC- and NLRP3-independent

We next examined the impact of ASC and NLRP3 deficiency on the host cytokine response to $S$. Typhimurium in the typhoid mouse model. We measured concentrations of TNF- $\alpha$, IFN- $\gamma$, MCP-1, IL-6, IL-10 and IL-12p70 in plasma of $\mathrm{Asc}^{-/-}, \mathrm{Nlrp3}^{-/-}$and WT mice 2 and 5 days after infection. Two days post-infection most systemic cytokine levels were still below the detection limit (data not shown). After 5 days of infection, however, plasma levels TNF- $\alpha$, monocyte chemo attractive protein (MCP)-1, IL-6, IL-10 and IL-12 were markedly elevated in all WT mice (Figure 2). Remarkably, neither ASC nor NLRP3 deficiency influenced systemic inflammation: no differences were found in any of the cytokine levels measured when comparing WT with $A s c^{-/-}$or $\mathrm{Nlrp}^{-/-}$mice, except for a slight increase in IL-12 in $\mathrm{Asc}^{-/-}$mice 5 days post-infection which could be a compensation for the lack of IL-18 observed in these mice. Surprisingly, plasma IFN- $\gamma$ levels (Figure 2B) did not differ between WT and $A s c^{-1-}$ mice during $S$. Typhimurium infection (2 and 5 days post-infection) despite the observed marked differences in release of IL-18, which is a key trigger for IFN- $\gamma$ release via NK and T-cells, dendritic and other phagocytic cells during Salmonella infection [14,15].

\section{Severe hepatic inflammation and distant organ injury during $S$. Typhimurium infection are ASC- and NLRP3-independent}

Next, we semi-quantitatively scored for the extent of inflammation histological samples of liver and spleen obtained 2 and 5 days after infection to further evaluate the role of ASC and NLRP3 in systemic in vivo host defense against $S$. Typhimurium in the typhoid model. During $S$. Typhimurium infection, hepatic inflammation was characterized by significant inflammation with necrosis, abscess formation, portal inflammation and thrombus formation, which was most prominent 5 days after infection (Figure 3). No differences were observed between WT, $\mathrm{Asc}^{-/-}$and $\mathrm{Nlrp}^{-/-}$mice (Figure 3) in pathology scores, which corresponded to the results of bacterial burdens and cytokine profiles. Spleen inflammation scores were similar in all three mice strains studied at all time points (data not shown). Consistent with these pathology data, systemic markers of organ injury were significantly 


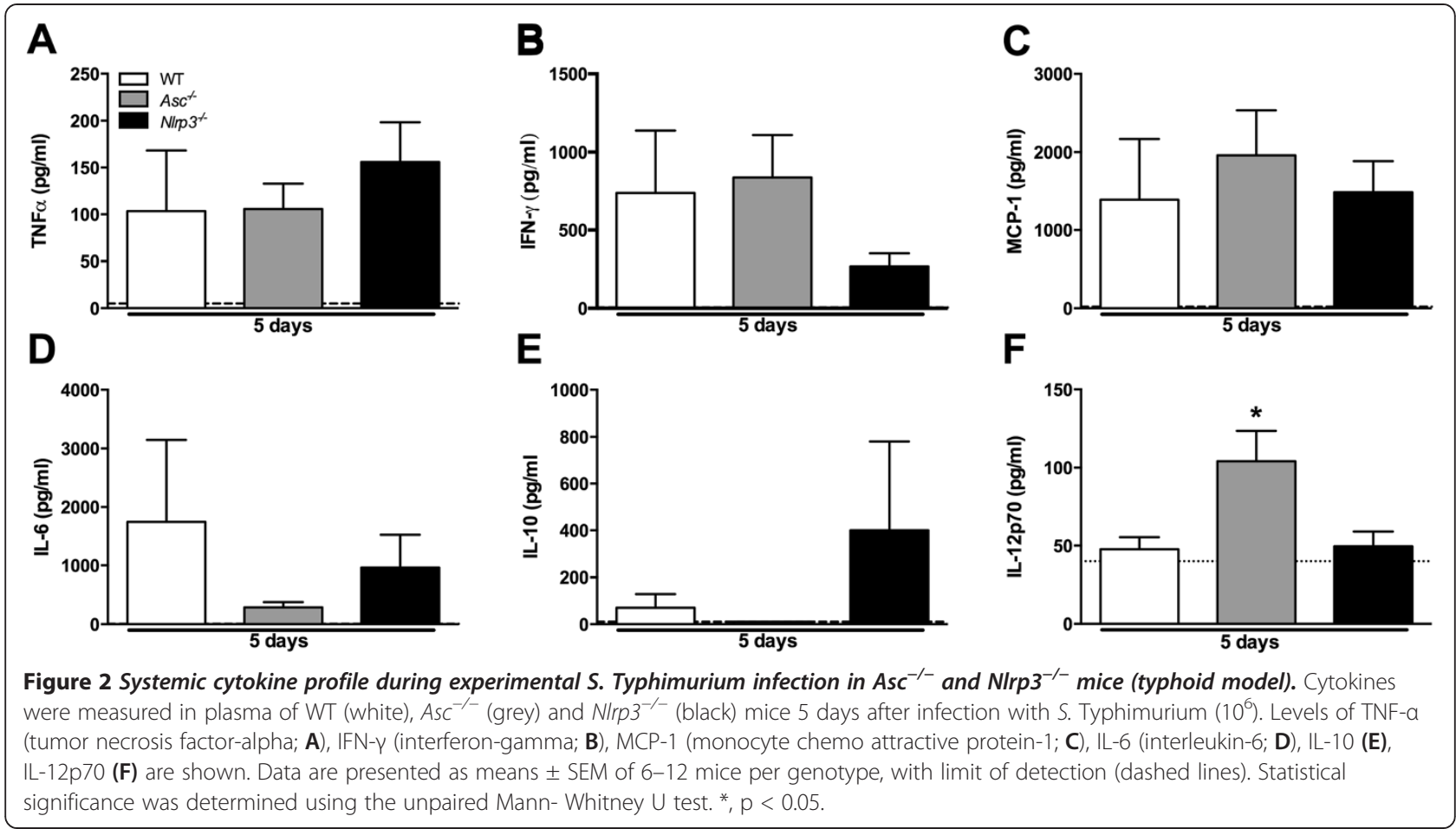

elevated during $S$. Typhimurium infection, reflecting increased hepatocellular injury (indicated by elevated levels of Aspartate aminotransferase (AST) and alanine transaminase (ALT)) and tissue damage (indicated by elevated lactate dehydrogenase (LDH) levels). However, these effects were not influenced by ASC or NLRP3 deficiency (Figure 4).

\section{Limited role for both ASC and NLRP3 in systemic host defense against $S$. Typhimurium in the colitis model} From the previous studies, which were performed in the typhoid model of Salmonella infection, we concluded that ASC and NLRP3 only play a very limited role in the systemic immune response to $S$. Typhimurium infection. To investigate whether these findings were dependent on the model chosen, we examined the role of these inflammasome components in the colitis model of Salmonella infection, in which mice were pre-treated orally with streptomycin. Similar to the typhoid model, $\mathrm{Asc}^{-1-}$ mice displayed undetectable IL-18 levels, whereas plasma IL-18 concentrations in $\mathrm{Nlrp}^{-/-}$mice did not differ from those in WT mice (Figure 5A). In contrast to the typhoid model, wherein mice do not develop marked inflammation of the cecum and colon, the colitis model results in a profound local inflammatory response with severe colitis and abscess formation after which Salmonella spreads systemically (Additional file 1). We found no difference when comparing WT, $\mathrm{Asc}^{-/-}$and $\mathrm{Nlrp3^{-/- }}$ mice in our colitis model with respect to bacterial counts and dissemination towards the liver and the systemic compartment 4 days after infection (Figure 5B-D), which corresponds to our findings in the typhoid model; the only exception being a slight increase in bacterial counts in the MLNs of $\mathrm{Asc}^{-/-}$ mice when compared to MLNs from WT mice. No differences were observed in plasma TNF- $\alpha$, MCP-1, IL-6, IL-10 and IL-12 levels between WT, $\mathrm{Asc}^{-/-}$and $\mathrm{Nlrp3}^{-/-}$ mice after infection with $S$. Typhimurium (Figure 6). Of note, in the colitis model, IFN- $\gamma$ correlated to IL-18 production and was reduced in the $\mathrm{Asc}^{-1-}$ mice when compared to WT mice $(234 \pm 66$ to $1831 \pm 426 \mathrm{pg} / \mathrm{ml}$, $\mathrm{p}=0.001$; Figure 6B). A difference between liver and spleen pathology is seen when comparing the typhoid and colitis models: while the typhoid fever model is characterized by marked hepatic necrosis, this was not regularly observed in the colitis model, while marked hepatic inflammatory cell influx is seen in both models (Figure 5J-L) Moreover, in the colitis model Nlrp3 $3^{-/-}$ (and to a lesser extent, $A s c^{-/-}$mice) displayed slightly increased spleen and liver parenchyma inflammation with corresponding elevated AST, albeit not ALT, levels when compared to WT mice. This may suggest that the differences in inflammation or damage caused by $S$. Typhimurium may be IL-18 independent.

\section{Discussion}

In the present study we aimed to characterize the in vivo relevance of the central inflammasome molecules ASC and NLRP3 in two different murine models of 


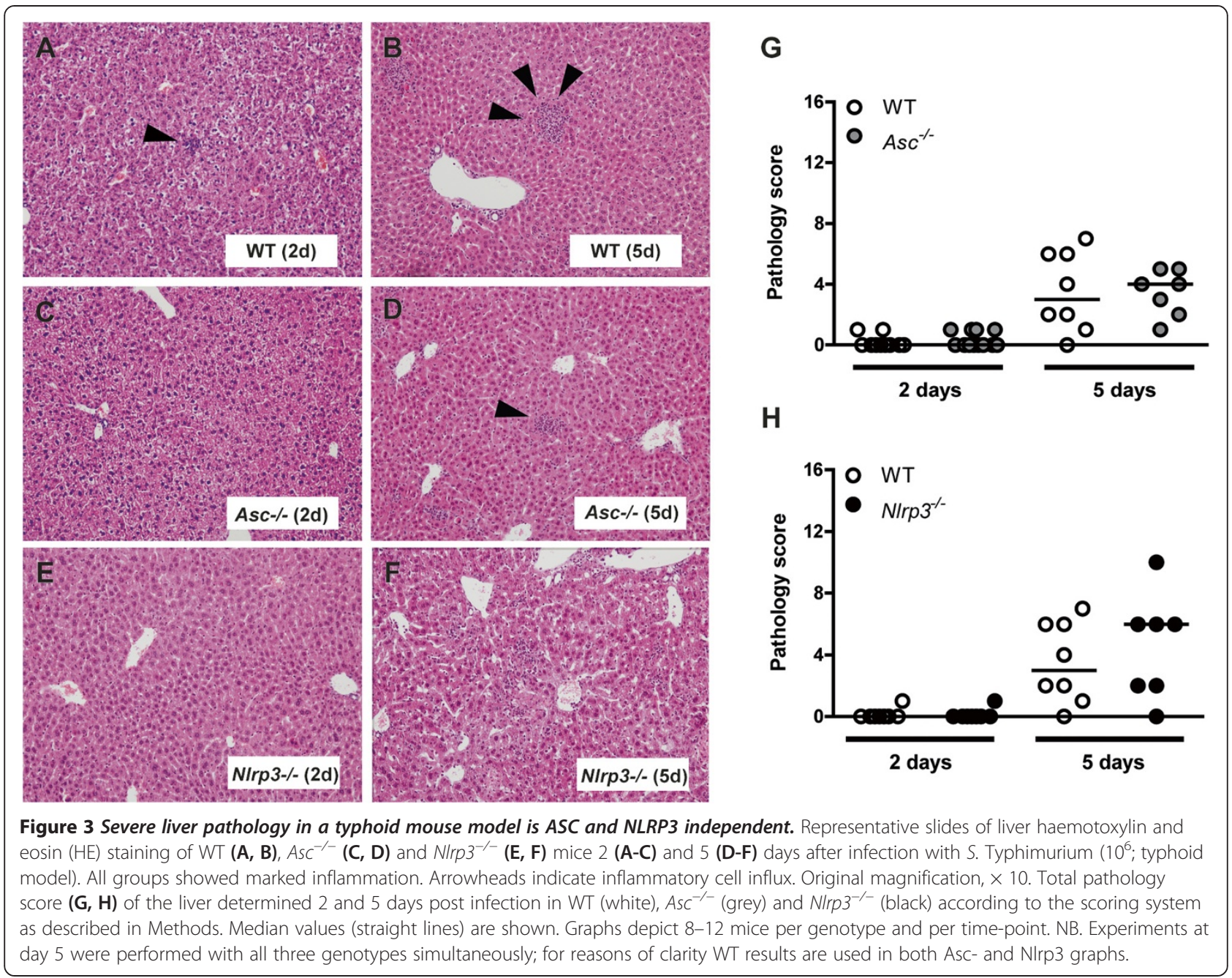

systemic Salmonella infection. Although in vitro studies confirmed that the inflammasome is activated during $S$. Typhimurium infection, we here show that ASC and NLRP3 individually are dispensable for the development of innate immunity against the pathogen. In the murine typhoid model, in which mice were not pretreated with streptomycin prior to oral inoculation with $S$. Typhimurium, equal bacterial counts were seen in WT, $\mathrm{Asc}^{-/-}$and $\mathrm{Nlrp3}^{-/-}$mice after infection in all organs (MLNs, liver, spleen, blood) at all time points. In line with the bacterial loads, proinflammatory cytokine levels, markers for tissue damage and organ pathology did not differ between groups. These results were replicated in a colitis model of $S$. Typhimurium infection, in which mice were pretreated with streptomycin prior to oral inoculation, were we saw an equally limited role for both ASC and NLRP3 in systemic host defense against $S$. Typhimurium. Taken together, the present results reveal a surprisingly limited role for ASC and NLRP3 during in vivo $S$. Typhimurium infection when dissecting different local and systemic compartments, especially given the fact that Salmonella infection is generally believed to be intracellular and that the inflammasome has previously been described to be crucial for the host defense against other intracellular pathogens [9].

NLRP3 can be triggered via terminal signals such as lysosomal rupture and the release of cathepsins and potassium, and the production of reactive oxygen species. The precise bacterial trigger for NLRP3 in S. Typhimurium infection remains unclear, since it has been demonstrated that Salmonella pathogenicity island-2 T3SS mutants (which lack the capacity to replicate intracellularly) are still detected by NLRP3 $[9,18,28]$. More recently, it was shown that NLRP3 inflammasome activation is bacterial RNA-mediated [29], which might explain the NLRP3-dependent sensing of these mutants. Viable $S$. Typhimurium can trigger both the NRLP3 and NLRC4 inflammasomes in vitro, resulting in activation of caspase-1, without involvement of other inflammasomes. The release of IL- $1 \beta$ was shown to be 

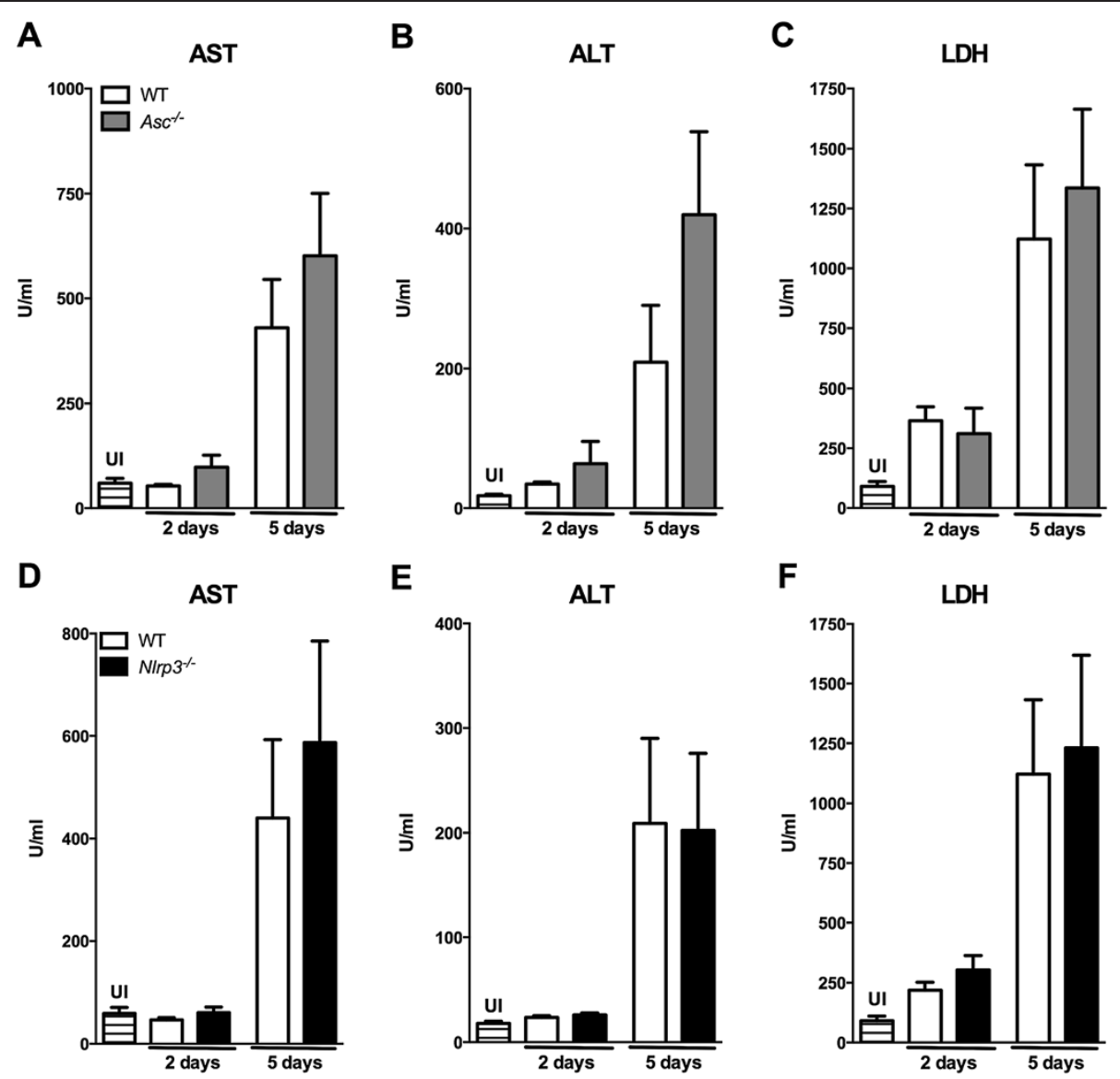

Figure 4 In a typhoid mouse model markers of hepatocellular injury are equally enhanced in all genotypes. Aspartate transaminase (AST), alanine transaminase (ALT) and lactate dehydrogenase (LDH), measured in blood plasma in WT (white), Asc ${ }^{-1-}$ (grey) (A-C) and Nlrp3 $3^{-/-}$(black) (D-F) mice after oral infection with S. Typhimurium $\left(10^{6}\right.$; typhoid model). UI, uninfected controls (striped). In all charts the mean and SEM is shown. Graphs depict 8-12 mice per genotype and per time-point. NB. Experiments at day 5 were performed with all three genotypes simultaneously; for reasons of clarity WT results are used in both Asc- and Nlrp3 graphs.

completely dependent on the combined function of the NLRP3 and NLRC4 inflammasomes and blocking either one lead to partially decreased IL-1 $\beta$ release [18]. The adaptor molecule ASC is a crucial component of both the NLRP3 and NLRC4 inflammasomes as it can bridge the pyrin and CARD domain of these NLRs to caspase-1, which is necessary for its activation [30]. $\mathrm{Asc}^{-/-}$bone-marrow derived macrophages stimulated with $S$. Typhimurium in vitro had defects in both the NLRP3 and NLRC4 inflammasomes with regard to releasing mature IL-1 $\beta$ [18]. Although these studies all underscore the potential role for the inflammasome during Salmonella infection in vitro, previous in vivo studies were not as evident.

During $S$. Typhimurium infection IL-1 $\beta$ is reported to be critical for the intestinal phase of the disease, while IL-18 is important for resistance to systemic infection but not the early gastrointestinal phase of infection [19]. Mice lacking both Nlrp3 and Nlrc4 genes showed an increased susceptibility to $S$. Typhimurium infection, similar to caspase- $1^{-/-}$or $\mathrm{Il}-18^{-/-}$mice, whereas mice lacking either NLRP3 or NLRC4 did not succumb earlier to infection, which was ascribed to the ability of NLRP3 and NLRC4 to be engaged by distinct signals $[18,19,21]$. In previous studies using a typhoid infection model, $A \mathrm{Sc}^{-/-}$mice were shown to have comparable bacterial loads (MLNs, spleen and liver) relative to WT mice when challenged with a different Salmonella strain (S. Typhimurium SL1344), while their serum IL-18 levels remained low, albeit not significantly different $[18,21]$. It was therefore speculated that ASC may play a role in vivo in the maturation of cytokines, whereas it is not essential for restricting bacterial growth, suggesting that additional ASC-independent pathways could be involved in activating caspase- 1 in response to NLRC4 and NLRP3 activation [18]. Indeed, it was shown that NLRC4 could also activate caspase- 1 even in the absence of ASC, to induce pyroptosis, ASC however remains to be crucial for 


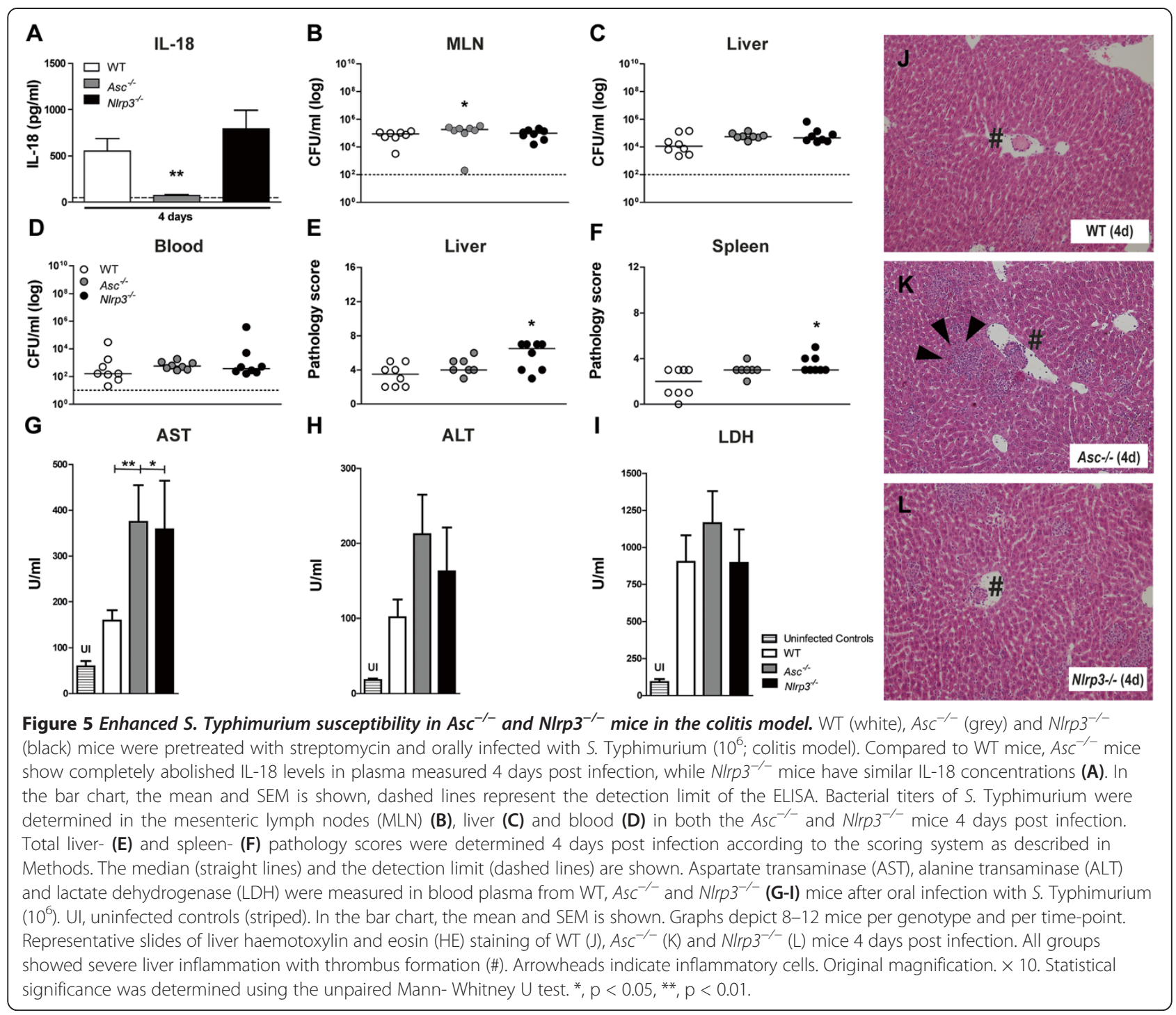

cytokine maturation during Salmonella infection [17,22]. Furthermore, intracellular Salmonella could induce a form of lytic cell death via caspase-11 [20], which was IFN- $\gamma$ mediated $[15,31]$. To study the in vivo role of the ASC and NLRP3 molecules in more depth we here made use of two different lethal $S$. Typhimurium models modeling typhoid and colitis disease. We showed that IL-18 release in plasma of $\mathrm{Asc}^{-/-}$mice was significantly decreased in both models and previous studies have suggested that impaired IL-18 release could enhance $S$. Typhimurium susceptibility in mice [19]. Surprisingly, we were unable to find a role for either ASC or NLRP3 in the host defense against $S$. Typhimurium infection in the typhoid model: no differences were found in bacterial counts in any organ (MLNs, liver, spleen and blood) at all time points. Furthermore, proinflammatory cytokine levels (TNF- $\alpha$, IL-6, IL-10, MCP-1, IFN- $\gamma$ ), markers for hepatocellular injury (AST, ALT), cell damage in general (LDH) and organ pathology (liver, spleen) did not differ between groups. In a similar study using a Burkholderia pseudomallei infection model, it was noted that IL-18 release was drastically reduced in $\mathrm{Asc}^{-/-}$ and $\mathrm{Nlrp}^{-/-}$mice, although it was still detectable in these mice at higher levels than uninfected mice, leading to the conclusion that inflammasome-independent production of IL-18 may be sufficient to provide some level of protection against infection with low dosages of Burkholderia pseudomallei [32]. This could be an explanation for the phenotype observed in the $\mathrm{Nlrp}^{-/-}$mice, which was comparable to WT mice, but not for the $\mathrm{Asc}^{-/-}$mice, as in both our infection models the IL-18 levels of $\mathrm{Asc}^{-/-}$ mice remained below detection limit independent of the stage of infection.

Although no differences were observed between bacterial loads in WT and $\mathrm{Asc}^{-/-}$or $\mathrm{Nlrp3}^{-/-}$mice in the 


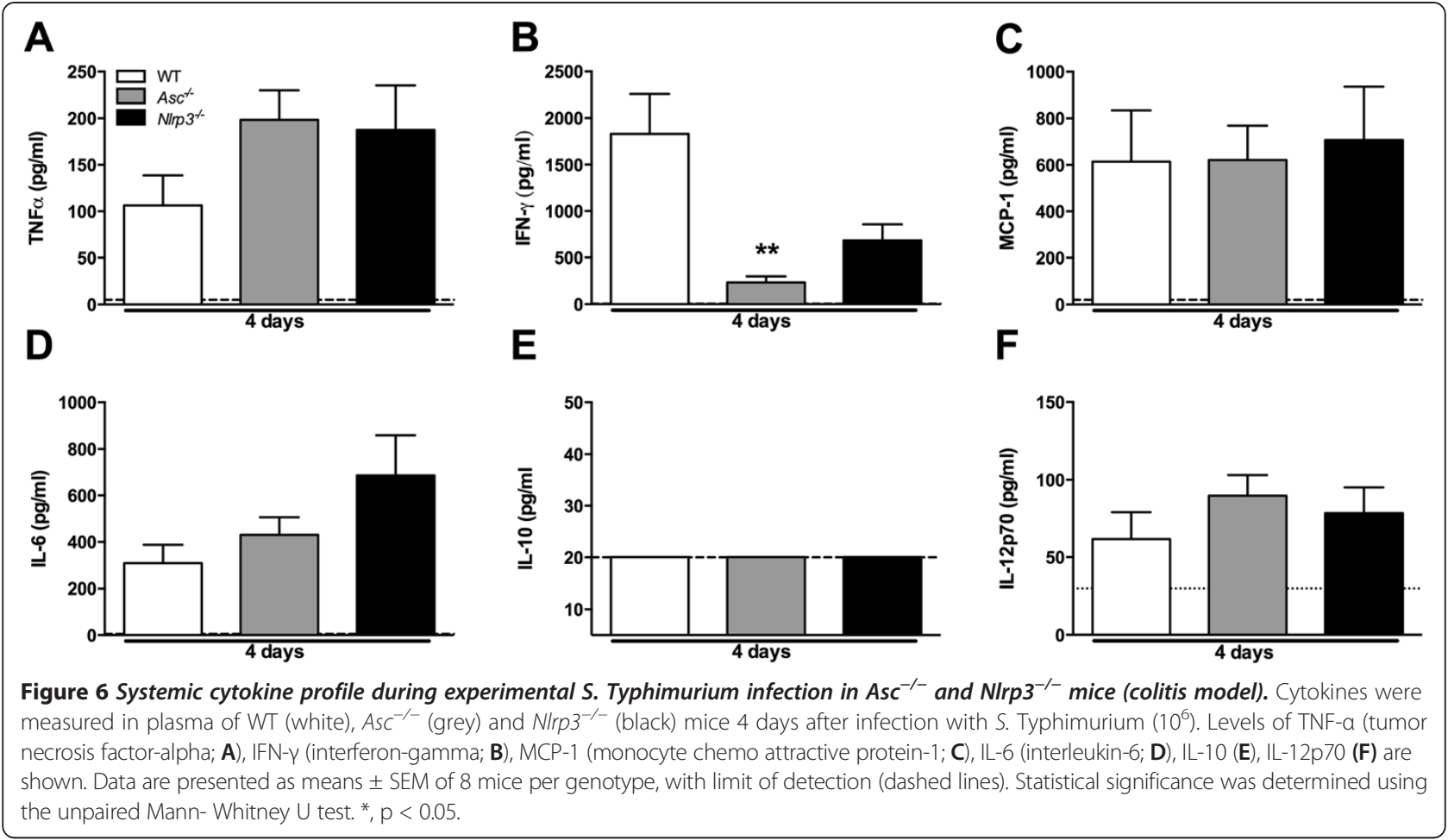

typhoid model, small differences in the streptomycin pre-treated group were seen with regard to bacterial loads (MLN) and splenic and hepatic inflammation between groups. A potential explanation for the different roles played by ASC in both models with regards to hepatocellular injury could be the marked difference in IFN- $\gamma$ release: where IFN- $\gamma$ was not reduced in the typhoid model, it was reduced in $\mathrm{Asc}^{-/-}$mice in the colitis model. In Salmonella-infected mice pre-treated with streptomycin, IFN- $\gamma$ seems to coordinate T-cell responses and the anti-microbial activity of phagocytes [33]. $\mathrm{Il}-18^{-/-}$mice show an increased susceptibility to $S$. Typhimurium infection [19]. Interestingly, in vivo IL-18 neutralization causes a marked decrease in circulating IFN- $\gamma$ levels during Salmonella infection [16]. It could therefore be hypothesized that the increased susceptibility to $S$. Typhimurium of $\mathrm{Il}-18^{-/-}$mice could in part be caused by the low circulating IFN- $\gamma$ levels and not by absence of IL-18 per se. The normal IFN- $\gamma$ response in the typhoid model could potentially trigger the described ASC-independent pathway via caspase-11 and IFN- $\gamma$ [20]. It has to be noted, however, that this does not explain the observed differences between IFN- $\gamma$ levels in the typhoid- and colitis model in $\mathrm{Asc}^{-/-}$mice and the increased hepatocellular injury inflicted upon $\mathrm{Nlrp}^{-/-}$mice despite lower but not significantly different IFN- $\gamma$ levels in the colitis model.

Our study has several limitations. Pre-growth conditions of Salmonella could have influenced our results as it determines expression of SPI-1 and SPI-2 expression among other virulence factors necessary for inflammasome activation $[9,29]$. We choose to use log-phase bacteria in our models according to standard methods in order to induce experimental infection in mice. Furthermore, the NLRC4 receptor has been described to play an important role during Salmonella infection [18,34]; adding a functional knockout mice to our experimental protocol could have given additional insights into the role of this inflammasome receptor in the typhoid and colitis model. It should be noted however that at present 22 human NLRs have been described and 34 mouse NLRs [35]. A plausible alternative interpretation of our work might be the presence of notable redundancy in the mouse system of NLRs so that one or more NLRs may be able to take over the role of NLRP3 in knockout mice (but not necessarily in humans), and that a double (or triple) knockout may be necessary in order to see a phenotype (Asc/Il-18 double KO for instance). Indeed, Broz et al. recently showed that Nlrp3/Nlrc4 double knockout mice have a markedly increased susceptibility for invasive salmonellosis when compared to WT controls [18].

\section{Conclusions}

In this work, we tried to further detail the role of keyinflammasome proteins ASC and NLRP3 during in vivo Salmonella infection. Inflammasome pathways are intensely complex and have proven to show immense redundancy. Indeed, our results reveal a limited role for both ASC and NLRP3 during in vivo S. Typhimurium infection, 
which is remarkable given the central role attributed to the inflammasome in the host defense against intracellular bacteria. In the future, it would be of great interest to study these proteins in humans with invasive salmonellosis or to make use of $\mathrm{KO}$ mice with complete blockage of all inflammasomes important in Salmonella pathogenesis.

\section{Additional file}

Additional file 1: Severe intestinal pathology seen in mice pretreated with streptomycin post infection with S. Typhimurium. Wild-type (WT) mice were starved for $12 \mathrm{~h}$ (typhoid fever model) or pretreated with streptomycin (colitis model) before infection with S. Typhimurium $\left(10^{6}\right)$ per os and sacrificed post-infection to assess intestinal pathology. Representative photographs of haemotoxylin and eosin (HE) stained slides are displayed of the cecum of the typhoid model (A-C) and colitis model (D-F) 4-5 days post infection. Original magnifications. $\times 4$ (A, D), $\times 10(B, E)$, and $\times 20(C, F)$. Severe intestinal pathology characterized by neutrophil infiltration, edema and extensive destructive ulceration of the mucosa is seen in mice pretreated with streptomycin (colitis model) but not in a typhoid fever model. Arrowheads indicate severe colitis in mice pretreated with streptomycin accompanied by crypt abscesses $(*)$.

\section{Abbreviations}

NTS: Non-typhoidal Salmonella; PRR: Pattern recognition receptor; TLR: Tolllike receptor; S. enterica: Salmonella enterica; PRR: Pattern recognition receptor; TLR: Toll-like receptor; NLR: NOD-like receptor; IL: Interleukin; TNFa: Tumor necrosis factor-alpha; IFN- $\gamma$ : Interferon-gamma; T3SS: Type 3 secretion system; LPS: Lipopolysaccharide; NLRP3: NOD-like receptor pyrin domain containing 3; NLRC4: NOD-like receptor, CARD domain-containing protein 4; ASC: Apoptosis-associated speck-like protein containing a CARD; SPI: Salmonella pathogenicity island; WT: Wild-type; LB: Luria-broth; HBSS: Hank's buffered salt solution; BA: Blood-agar; MLN: Mesenteric lymph nodes; MCP-1: Monocyte chemo attractive protein-1; AST: Aspartate aminotransferase; ALT: Alanine transaminase; LDH: Lactate dehydrogenase.

\section{Competing interests}

The authors declare that they have no competing interests.

\section{Authors' contributions}

Conceived and designed the experiments: HdJ, JvD, TvdP, and JW. Performed the experiments: HdJ, GK and MvL. Analysed the data: HdJ, MvL, GK, JR, JW. Wrote the paper: HdJ, TvdP, JW. All authors approved the final version for submission.

\section{Acknowledgments \\ $\mathrm{HdJ}$ received unrestricted funding for this project from the Academic Medical Center (AMC PhD Scholarship). JW: The Netherlands Organisation for Scientific Research (NWO; VENI grant number 91610008) and The Netherlands Organisation for Health Research and Development (ZonMw; Clinical Fellowship grant number 90700424). We thank Richard A. Flavell, Yale University School of Medicine, for generously providing us the $\mathrm{Asc}^{-\prime}$ and Nlrp3 $3^{-1-}$ mouse strains. We also would like to thanks Marieke van den Brink and Joost Daalhuisen for technical assistance with mouse handling.}

\section{Author details}

'Department of Internal Medicine, Division of Infectious Diseases and Center for Infection and Immunity Amsterdam (CINIMA), Academic Medical Center, Meibergdreef 9, Room G2-132, 1105, AZ, Amsterdam, the Netherlands. ${ }^{2}$ Center for Experimental and Molecular Medicine (CEMM), Academic Medical Center, Meibergdreef 9, Room G2-132, 1105, AZ, Amsterdam, the Netherlands. ${ }^{3}$ Department of Infection and Tropical Medicine, Heartlands Hospital, Bordesley Green East, Birmingham B9 5SS, UK. ${ }^{4}$ Department of Pathology, Academic Medical Center, Meibergdreef 9, Room G2-132, 1105, AZ, Amsterdam, the Netherlands. ${ }^{5}$ Department of Infectious Diseases, Leiden University Medical Center, Box 9600, 2300, RC, Leiden, the Netherlands.
Received: 4 January 2014 Accepted: 18 July 2014

Published: 13 August 2014

\section{References}

1. Crump JA, Mintz ED: Global trends in typhoid and paratyphoid Fever. Clin Infect Dis 2010, 50(2):241-246.

2. Feasey NA, Dougan G, Kingsley RA, Heyderman RS, Gordon MA: Invasive non-typhoidal salmonella disease: an emerging and neglected tropical disease in Africa. Lancet 2012, 379(9835):2489-2499.

3. de Jong HK, Parry CM, van der Poll T, Wiersinga WJ: Host-pathogen interaction in invasive salmonellosis. PLoS Pathog 2012, 8(10):e1002933.

4. Dougan $G$, John V, Palmer S, Mastroeni P: Immunity to salmonellosis. Immunol Rev 2011, 240(1):196-210.

5. Parry CM, Hien TT, Dougan G, White NJ, Farrar JJ: Typhoid fever. N Engl J Med 2002, 347(22):1770-1782

6. Parry $\mathrm{CM}$, Threlfall EJ: Antimicrobial resistance in typhoidal and nontyphoidal salmonellae. Curr Opin Infect Dis 2008, 21(5):531-538.

7. Kawai T, Akira S: The role of pattern-recognition receptors in innate immunity: update on Toll-like receptors. Nat Immunol 2010, 11(5):373-384.

8. Schroder K, Tschopp J: The inflammasomes. Cell 2010, 140(6):821-832.

9. Broz P, Monack DM: Molecular mechanisms of inflammasome activation during microbial infections. Immunol Rev 2011, 243(1):174-190.

10. Butler T, Ho M, Acharya G, Tiwari M, Gallati H: Interleukin-6, gamma interferon, and tumor necrosis factor receptors in typhoid fever related to outcome of antimicrobial therapy. Antimicrob Agents Chemother 1993, 37(11):2418-2421.

11. Keuter M, Dharmana E, Gasem MH, van der Ven-Jongekrijg J, Djokomoeljanto R, Dolmans WM, Demacker P, Sauerwein R, Gallati H, van der Meer JW: Patterns of proinflammatory cytokines and inhibitors during typhoid fever. J Infect Dis 1994, 169(6):1306-1311.

12. Raffatellu M, Chessa D, Wilson RP, Tukel C, Akcelik M, Baumler AJ: Capsule-mediated immune evasion: a new hypothesis explaining aspects of typhoid fever pathogenesis. Infect Immun 2006, 74(1):19-27.

13. Thompson LJ, Dunstan SJ, Dolecek C, Perkins T, House D, Dougan G, Nguyen TH, Tran TP, Doan CD, Le TP, Nguyen TD, Tran TH, Farrar JJ, Monack D, Lynn DJ, Popper SJ, Falkow S: Transcriptional response in the peripheral blood of patients infected with Salmonella enterica serovar Typhi. Proc Natl Acad Sci U S A 2009, 106(52):22433-22438.

14. Monack DM, Bouley DM, Falkow S: Salmonella typhimurium persists within macrophages in the mesenteric lymph nodes of chronically infected Nramp1+/+mice and can be reactivated by IFNgamma neutralization. J Exp Med 2004, 199(2):231-241.

15. Kupz A, Guarda G, Gebhardt T, Sander LE, Short KR, Diavatopoulos DA, Wijburg OL, Cao H, Waithman JC, Chen W, Fernandez-Ruiz D, Whitney PG, Heath WR, Curtiss R, Tschopp J, Strugnell RA, Bedoui S: NLRC4 inflammasomes in dendritic cells regulate noncognate effector function by memory $\mathrm{CD} 8(+)$ T cells. Nat Immunol 2012, 13(2):162-169.

16. Mastroeni $\mathrm{P}$, Clare $\mathrm{S}$, Khan $\mathrm{S}$, Harrison JA, Hormaeche CE, Okamura $\mathrm{H}$, Kurimoto M, Dougan G: Interleukin 18 contributes to host resistance and gamma interferon production in mice infected with virulent Salmonella typhimurium. Infect Immun 1999, 67(2):478-483.

17. Miao EA, Leaf IA, Treuting PM, Mao DP, Dors M, Sarkar A, Warren SE, Wewers MD, Aderem A: Caspase-1-induced pyroptosis is an innate immune effector mechanism against intracellular bacteria. Nat Immunol 2010, 11(12):1136-1142.

18. Broz P, Newton K, Lamkanfi M, Mariathasan S, Dixit VM, Monack DM: Redundant roles for inflammasome receptors NLRP3 and NLRC4 in host defense against Salmonella. J Exp Med 2010, 207(8):1745-1755.

19. Raupach B, Peuschel SK, Monack DM, Zychlinsky A: Caspase-1-mediated activation of interleukin-1 beta (IL-1 beta) and IL-18 contributes to innate immune defenses against Salmonella enterica serovar Typhimurium infection. Infect Immun 2006, 74(8):4922-4926.

20. Broz P, Ruby T, Belhocine K, Bouley DM, Kayagaki N, Dixit VM, Monack DM: Caspase-11 increases susceptibility to Salmonella infection in the absence of caspase-1. Nature 2012, 490:288-291.

21. Lara-Tejero M, Sutterwala FS, Ogura Y, Grant EP, Bertin J, Coyle A, Flavell RA, Galan JE: Role of the caspase-1 inflammasome in Salmonella typhimurium pathogenesis. J Exp Med 2006, 203(6):1407-1412.

22. Broz P, von Moltke J, Jones JW, Vance RE, Monack DM: Differential requirement for Caspase-1 autoproteolysis in pathogen-induced cell death and cytokine processing. Cell Host Microbe 2010, 8(6):471-483. 
23. Sutterwala FS, Ogura Y, Szczepanik M, Lara-Tejero M, Lichtenberger GS, Grant EP, Bertin J, Coyle AJ, Galan JE, Askenase PW, Flavell RA: Critical role for NALP3/CIAS1/Cryopyrin in innate and adaptive immunity through its regulation of caspase-1. Immunity 2006, 24(3):317-327.

24. Carter PB, Collins FM: The route of enteric infection in normal mice. J Exp Med 1974, 139(5):1189-1203.

25. Barthel M, Hapfelmeier S, Quintanilla-Martinez L, Kremer M, Rohde M, Hogardt M, Pfeffer K, Russmann H, Hardt WD: Pretreatment of mice with streptomycin provides a Salmonella enterica serovar Typhimurium colitis model that allows analysis of both pathogen and host. Infect Immun 2003, 71(5):2839-2858.

26. Hapfelmeier S, Hardt WD: A mouse model for S. typhimurium-induced enterocolitis. Trends Microbiol 2005, 13(10):497-503.

27. Santos RL, Zhang S, Tsolis RM, Kingsley RA, Adams LG, Baumler AJ: Animal models of Salmonella infections: enteritis versus typhoid fever. Microbes Infect 2001, 3(14-15):1335-1344.

28. Kufer TA, Sansonetti PJ: NLR functions beyond pathogen recognition. Nat Immunol 2011, 12(2):121-128.

29. Sander LE, Davis MJ, Boekschoten MV, Amsen D, Dascher CC, Ryffel B, Swanson JA, Muller M, Blander JM: Detection of prokaryotic mRNA signifies microbial viability and promotes immunity. Nature 2011, 474(7351):385-389.

30. Martinon F, Mayor A, Tschopp J: The inflammasomes: guardians of the body. Annu Rev Immunol 2009, 27:229-265.

31. Mayer-Barber KD, Barber DL, Shenderov K, White SD, Wilson MS, Cheever A, Kugler D, Hieny S, Caspar P, Nunez G, Schlueter D, Flavell RA, Sutterwala FS, Sher A: Caspase-1 independent IL-1 beta production is critical for host resistance to mycobacterium tuberculosis and does not require TLR signaling in vivo. J Immunol 2010, 184(7):3326-3330.

32. Ceballos-Olvera I, Sahoo M, Miller MA, Del Barrio L, Re F: Inflammasome-dependent pyroptosis and IL-18 protect against Burkholderia pseudomallei lung infection while IL-1beta is deleterious. PLoS Pathog 2011, 7(12):e1002452.

33. Kaiser P, Diard M, Stecher B, Hardt WD: The streptomycin mouse model for Salmonella diarrhea: functional analysis of the microbiota, the pathogen's virulence factors, and the host's mucosal immune response. Immunol Rev 2012, 245(1):56-83.

34. Mariathasan S, Newton K, Monack DM, Vucic D, French DM, Lee WP, Roose-Girma M, Erickson S, Dixit VM: Differential activation of the inflammasome by caspase-1 adaptors ASC and Ipaf. Nature 2004, 430(6996):213-218.

35. Ting JP, Lovering RC, Alnemri ES, Bertin J, Boss JM, Davis BK, Flavell RA, Girardin SE, Godzik A, Harton JA, Hoffman HM, Hugot JP, Inohara N, Mackenzie A, Maltais $\sqcup$, Nunez G, Ogura Y, Otten LA, Philpott D, Reed JC, Reith W, Schreiber S, Steimle V, Ward PA: The NLR gene family: a standard nomenclature. Immunity 2008, 28(3):285-287.

doi:10.1186/s12865-014-0030-7

Cite this article as: De Jong et al: Limited role for ASC and NLRP3

during in vivo Salmonella Typhimurium infection. BMC Immunology 2014 15:30.

\section{Submit your next manuscript to BioMed Central and take full advantage of:}

- Convenient online submission

- Thorough peer review

- No space constraints or color figure charges

- Immediate publication on acceptance

- Inclusion in PubMed, CAS, Scopus and Google Scholar

- Research which is freely available for redistribution 